\title{
Networked Cooperative Distributed Model Predictive Control Based on State Observer
}

\author{
Baili Su, Yanan Zhao, Jinming Huang \\ College of Engineering, Qufu Normal University, Rizhao, China \\ Email: subaili111@126.com, ynz105@163.com, huangjm 163@163.com
}

Received 12 March 2016; accepted 26 June 2016; published 29 June 2016

Copyright (C) 2016 by authors and Scientific Research Publishing Inc.

This work is licensed under the Creative Commons Attribution International License (CC BY). http://creativecommons.org/licenses/by/4.0/

c) (i) Open Access

\begin{abstract}
Although distributed model predictive control has caused significant attention and received many good results, the results are mostly under the assumption that the system states can be observed. However, the states are difficult to be observed in practice. In this paper, a novel distributed model predictive control is proposed based on state observer for a kind of linear discrete-time systems where states are not measured. Firstly, an output feedback control law is designed based on Lyapunov function and state observer. And the stability domain is described. Furthermore, the stability domain as a terminal constraint is added into the constraint conditions of the algorithm to make systems stable outside the stability domain. The simulation results show the effectiveness of the proposed method.
\end{abstract}

\section{Keywords}

Distributed System, Model Predictive Control, Lyapunov Function, State Observer, Stable Domain, Cooperative Control

\section{Introduction}

In industrial processes, there exists a class of hybrid systems which are comprised of some subsystems which couple each other through energy, quality, etc. For example, urban drainage network system, transportation system, energy power, Net system and irrigation system. These systems have many components, wide space distribution, many constraints and many targets. We can obtain good control performance if the centralized control is used to control this kind of systems. But its flexibility and fault tolerance are relatively weak. If the distributed control is adopted, its flexibility and fault tolerance are better [1] [2]. So, the problem of distributed control for these hybrid systems has become an important research project [3] [4].

Model predictive control (MPC) is receding horizon control which can deal with the constraints of systems 
states and inputs during the design of optimization control [5]. It adopts the strategies such as feedback correction, rolling optimization [6] and has strong ability to deal with constraints and good dynamic performance [7]. Therefore, it can be more effective to solve the optimal control problem for distributed systems. That is distributed model predictive control [8].

In recent years, the research on the distributed predictive control method has developed greatly. There have been many beneficial results about it. In Literature [9], based on the research about the Nash optimal distributed predictive control, a networked predictive control strategy is proposed for series connection structure systems with network information mode whose subsystems are coupled each other. In Literature [10], for a class of linear systems with input-output constraints, a design method of stabilization distributed predictive control is given. But every subsystem's controller can only optimize this subsystem's performance index. The optimization method for the whole system's performance index is not given. In Literature [11], an iterative algorithm of stabilization controller with constrained input is designed. This method can optimize the overall performance of the system. However, it is necessary to obtain global information, which greatly reduces the flexibility and fault tolerance of the system. Literature [12] proposes a new distributed predictive coordinated control strategy to improve the performance of the whole system without increasing the connectivity degree. These references are obtained on the assumption that the system states can be measured.

However, in the actual application, the limitation of measuring equipment in economy makes the state feedback hard to realize. In reference [13], a distributed predictive control algorithm is designed in the case of the states not being measured. But this method can only optimize the performance of each subsystem, not the overall performance of the system.

In this paper, a distributed predictive control method based on Lyapunov function and state observer is designed to optimize the overall system's performance. This algorithm adds the quadratic function of the decoy system's input variables to the performance index of the subsystem, expands the coordination degree, and optimizes the performance of the system.

This paper is arranged as follows. In the second section, the control problem for distributed system under network mode is described in detail. The output feedback controller based on Lyapunov functions and state observers is designed in the third section, and the stability domain is given. The fourth section designs distributed predictive controller. In the fifth section, the distributed prediction controllers performance is analyzed, and the steps of the algorithm design are given. The simulation results verify the effectiveness of the method proposed in this paper in the sixth section. Conclusion is given in Section 7.

\section{Problem Formulation}

Consider the distributed system $S$ which is comprised of m related subsystems $S_{i}$. The state space description of $S_{i}$ can be expressed as

$$
\begin{aligned}
& x_{i}(k+1)=A_{i i} x_{i}(k)+B_{i i} u_{i}(k)+\sum_{j=1(j \neq i)}^{m} A_{i j} x_{j}(k)+\sum_{j=1(j \neq i)}^{m} B_{i j} u_{j}(k) \\
& y_{i}(k)=C_{i i} x_{i}(k)+\sum_{j=1(j \neq i)}^{m} C_{i j} x_{j}(k) \quad i=1, \cdots, m
\end{aligned}
$$

where $x_{i}(k) \in \mathbf{R}^{n_{x_{i}}}$ denotes the state variable of subsystem $S_{i}, \quad u_{i}(k) \in \mathbf{R}^{n_{u_{i}}}$ denotes the input variable and satisfies $\left|u_{i}(k)\right| \leq\left|u_{i \max }\right|$, and $y_{i}(k) \in \mathbf{R}^{n_{y_{i}}}$ denotes the measurable output variable. $A_{i i}, B_{i j}, A_{i j}, B_{i j}$ are constant matrices with corresponding dimension, respectively. The distributed structure of the system under the network mode is shown in Figure 1.

Synthesizing all subsystems, we can get the system model as:

$$
\begin{aligned}
& x(k+1)=A x(k)+B u(k) \\
& y(k)=C x(k)
\end{aligned}
$$

where, 


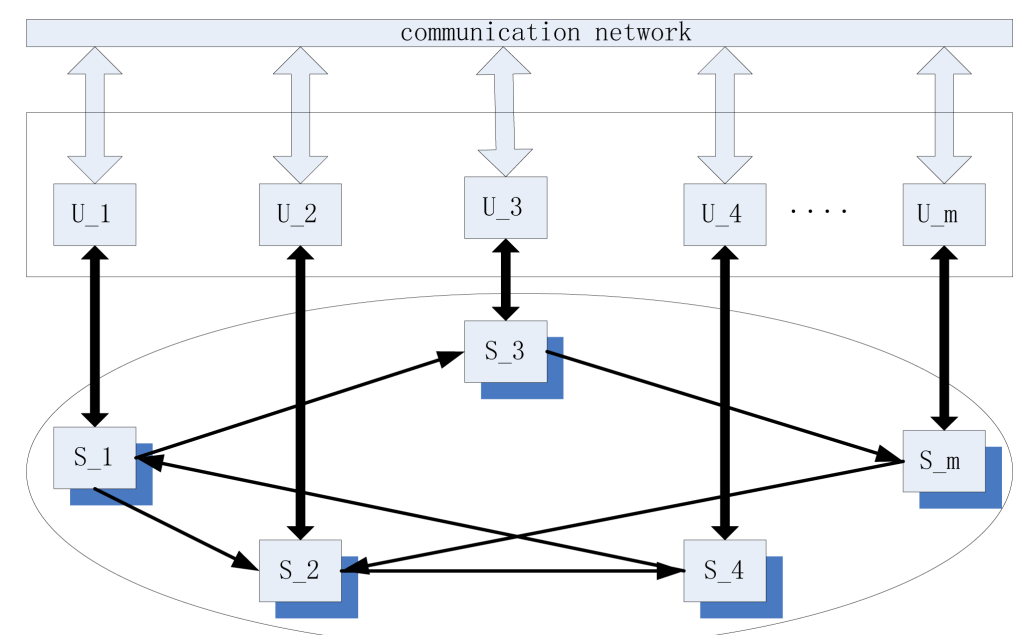

Figure 1. Distributed schematic diagram of the system under network pattern.

$$
\begin{aligned}
& A=\left[\begin{array}{ccc}
A_{11} & \cdots & A_{1 m} \\
\vdots & \ddots & \vdots \\
A_{m 1} & \cdots & A_{m m}
\end{array}\right] \quad B=\left[\begin{array}{ccc}
B_{11} & \cdots & B_{1 m} \\
\vdots & \ddots & \vdots \\
B_{m 1} & \cdots & B_{m m}
\end{array}\right] \quad C=\left[\begin{array}{ccc}
C_{11} & \cdots & C_{1 m} \\
\vdots & \ddots & \vdots \\
C_{m 1} & \cdots & C_{m m}
\end{array}\right] \\
& x(k)=\left[\begin{array}{llll}
x_{1}^{\mathrm{T}}(k) & x_{2}^{\mathrm{T}}(k) & \cdots & x_{m}^{\mathrm{T}}(k)
\end{array}\right]^{\mathrm{T}} \\
& u(k)=\left[\begin{array}{llll}
u_{1}^{\mathrm{T}}(k) & u_{2}^{\mathrm{T}}(k) & \cdots & u_{m}^{\mathrm{T}}(k)
\end{array}\right]^{\mathrm{T}} \\
& y(k)=\left[\begin{array}{llll}
y_{1}^{\mathrm{T}}(k) & y_{2}^{\mathrm{T}}(k) & \cdots & y_{m}^{\mathrm{T}}(k)
\end{array}\right]^{\mathrm{T}}
\end{aligned}
$$

The control objective is to design an output feedback control law for the linear discrete-time distributed system (3) (4) based on Lyapunov function and state observer under the premise that network connectivity and fault tolerance of the system are not added. And then the stability domain is described. Furthermore, taking the stability domain as a terminal constraint to design output feedback model predictive controller in order to make the system stable outside the stability domain. Make sure that under the premise of initial feasibility, the system is successive feasible.

\section{Output Feedback Control Based on Lypunov Function and State Observer}

This section shows the controller design based on Lypunov function under the states are available at first to get the stability domain description. Then it shows the output feedback controller design under the states are not available.

\subsection{The State-Feedback Controller Design Based on Lypunov Function}

Consider the subsystem (1) (2), and structure the state feedback controller as follows:

$$
u_{i}(k)=K_{i} x_{i}(k)
$$

where $K_{i}$ is the state feedback gain. Give the following assumption:

Assumption (i). For the subsystem $\mathcal{S}_{i}$, there exists feedback law $u_{i}(k)=K_{i} x_{i}(k)$ so that the eigenvalues of $A_{d_{i}}=A_{i i}+B_{i i} K_{i}$ are always in the unit circle, and the system $x(k+1)=A_{c} x(k)$ is asymptotically stable, where $A_{c}=A+B K, K=\operatorname{diag}\left\{K_{1}, K_{2}, \cdots, K_{m}\right\}$.

Define the following matrices:

$$
A_{d}=\operatorname{diag}\left\{A_{d 1}, A_{d 2}, \cdots, A_{d m}\right\}
$$




$$
A_{o}=A_{c}-A_{d}
$$

satisfy:

$$
A_{o}^{\mathrm{T}} P A_{o}+A_{o}^{\mathrm{T}} P A_{d}+A_{d}^{\mathrm{T}} P A_{o}<\frac{\hat{Q}}{2}
$$

where

$$
\hat{Q}=Q+K^{\mathrm{T}} R K>0
$$

and

$$
A_{d}^{\mathrm{T}} P A_{d}-P=-\hat{Q}
$$

where $Q, P, R$ are positive diagonal matrices, and $Q=\operatorname{diag}\left\{Q_{1}, Q_{2}, \cdots, Q_{m}\right\}, \quad P=\operatorname{diag}\left\{P_{1}, P_{2}, \cdots, P_{m}\right\}$, $R=\operatorname{diag}\left\{R_{1}, R_{2}, \cdots, R_{m}\right\}$

Lemma 1. If the Assumption (i) is satisfied, there exists a non-empty set $\Omega\left(c_{1}\right)=\left\{x \in X: x^{\mathrm{T}}(k) P x(k) \leq c_{1}, c_{1}>0\right\}$ as a invariant set of the system $x(k+1)=A_{c} x(k)$, and the system is stable under the state feedback control law $u(k)=K x(k)$, where $c_{1}$ is the biggest to make sure $c_{1} K P^{-1} K^{\mathrm{T}} \leq\left(u_{\max }\right)^{2}$.

proof. Select a Lyapunov function candidate $V=x^{\mathrm{T}}(k) P x(k)$.

The difference of $V(k)$ along the trajectories of the closed-loop system $x(k+1)=A_{c} x(k)$ is given by

$$
\begin{aligned}
\Delta V(k) & =V(k+1)-V(k) \\
& =x(k+1)^{\mathrm{T}} P x(k+1)-x(k)^{\mathrm{T}} P x(k) \\
& =x(k)^{\mathrm{T}}\left(A_{c}^{\mathrm{T}} P A_{c}-P\right) x(k) \\
& =x(k)^{\mathrm{T}}\left(A_{d}^{\mathrm{T}} P A_{d}-P+A_{o}^{\mathrm{T}} P A_{o}+A_{o}^{\mathrm{T}} P A_{d}+A_{d}^{\mathrm{T}} P A_{o}\right) x(k) \\
& <-\frac{1}{2} x(k)^{\mathrm{T}} \hat{Q} x(k)
\end{aligned}
$$

Since $\hat{Q}>0$, so $\Delta V(k)<0$.

Since the input constraint $|u(k)| \leq\left|u_{\max }\right|$, we have $|u(k)|^{2} \leq\left|u_{\max }\right|^{2}$.

And since $u(k)=K x(k)$, so $c_{1} K P^{-1} K^{\mathrm{T}} \leq\left|u_{\max }\right|^{2}$.

The proof is completed, and the set $\Omega$ is the invariant set of the system.

Therefore, all states from $\Omega\left(c_{1}\right)$ can always keep in $\Omega\left(c_{1}\right)$ and asymptotically stable at the origin. That is to say for the given positive real number $d$, if $x(0) \in \Omega\left(c_{1}\right)$, we have $x(k) \in \Omega\left(c_{1}\right)$, and

$$
\lim _{k \rightarrow \infty} \sup \|x(k)\| \leq d
$$

Thus, the stability domain of the subsystem $\mathcal{S}_{i}$ is defined as follows:

$$
\Omega_{i}\left(c_{1}\right)=\left\{x_{i} \in X_{i}: x_{i}^{\mathrm{T}}(k) P_{i} x_{i}(k) \leq c_{1}, c_{1}>0\right\}
$$

Suppose that at $k_{0}$, all states of the subsystem satisfy $x_{i}\left(k_{0}\right) \in \Omega_{i}\left(c_{1}\right)$, and the subsystems $\mathcal{S}_{i}$ use control law $K_{i} x_{i}$, so the system is asymptotically stable based on Lemma 1 .

\subsection{Output Feedback Controller Design Based on the State Estimation}

Design the state observer as follows [14]:

$$
\begin{aligned}
& \hat{x}(k+1)=A \hat{x}(k)+B u(k)+F(y(k)-C \hat{x}(k)) \\
& \hat{u}(k)=K \hat{x}(k)
\end{aligned}
$$

where $\hat{x}(k)$ is the observer state of the system, $F$ is the state observer gain to be identified. We can get the error dynamic equation based on Equation (3) and (5) as 


$$
\begin{aligned}
e(k+1) & =x(k+1)-\hat{x}(k+1) \\
& =A x(k)+B u(k)-A \hat{x}(k)-B u(k)-F(C x(k)-C \hat{x}(k)) \\
& =(A-F C) e(k)
\end{aligned}
$$

Therefore, the error dynamic equation of the state observer is regarded as a new autonomous system. That is to say if the new system (7) is stable, the estimation states can track the real states well.

Define a quadratic function on the observe error as follows:

$$
E(e(k))=e(k)^{\mathrm{T}} \bar{P} e(k)
$$

where, $\bar{P}=\bar{P}^{\mathrm{T}}>0$.

Theorem 1. Consider the error dynamic equation of the state observer (7), if there exist matrices $\bar{P}=\bar{P}^{\mathrm{T}}>0$ and $Y=\bar{P} F$, and the inequality

$$
\left[\begin{array}{cc}
\xi^{2} \bar{P}-L & (\bar{P} A-Y C)^{\mathrm{T}} \\
\bar{P} A-Y C & \bar{P}
\end{array}\right]>0
$$

is satisfied, then the inequality

$$
E(e(k+l+1))-\xi^{2} E(e(k+l))<-e(k+l)^{\mathrm{T}} L e(k+l)
$$

is satisfied, where $l=1,2,3, \cdots, \xi \in(0,1)$ are decay factors, $L$ is positive definite symmetric matrix, and satisfies $\xi^{2} \bar{P}-L>0$. So there exists $T>0$, such that if the inequality above is satisfied, then $e(k) \rightarrow 0$. In other words, the observer state $\hat{x}(k)$ converges to the real state $x(k)$.

Proof. By the Schur complement lemma, the inequality (8) is equivalent to

$$
\left(\xi^{2} \bar{P}-L\right)-(\bar{P} A-Y C)^{\mathrm{T}} \bar{P}^{-1}(\bar{P} A-Y C)>0
$$

Substituting $Y=\bar{P} F \quad$ into the above formula, we derive

$$
\xi^{2} \bar{P}-L-(A-F C)^{\mathrm{T}} \bar{P}(A-F C)>0
$$

Multiply $e(k+l)^{\mathrm{T}}$ and $e(k+l), l=1,2, \cdots$ in the both side of the above formula at the same time, we have

$$
\xi^{2} e(k+l)^{\mathrm{T}} \bar{P} e(k+l)-e(k+l)^{\mathrm{T}} L e(k+l)-e(k+l)^{\mathrm{T}}(A-F C)^{\mathrm{T}} \bar{P}(A-F C) e(k+l)>0
$$

By (8), we have

$$
\xi^{2} e(k+l)^{\mathrm{T}} \bar{P} e(k+l)-e(k+l)^{\mathrm{T}} L e(k+l)-e(k+l+1)^{\mathrm{T}} \bar{P} e(k+l+1)>0
$$

the inequality is satisfied.

Therefore, $E(e(k))$ is regarded as the Lyapunov function of zero input dynamic error system, and satisfies the stability constraints (9), then the autonomous system (7) is asymptotically stable. In other words, there always exists $T>0$, when $k>T, e(k) \rightarrow 0$, and observer state $\hat{x}(k)$ ultimately converge to the real state $x(k)$.

Remark 1. By Theorem 1, the state observer gain $F$ can be computed off line through the feasibility of the linear matrix inequality (8).

Thus, for the given $\Omega\left(c_{1}\right)$, if $x(0) \in \Omega\left(c_{1}\right)$, and $\hat{x}(0)^{\mathrm{T}} P x(0) \leq c$, then the closed loop system is asymptotically stable at the origin. There exists $d>0$, such that $\lim _{t \rightarrow \infty} \sup \|x(t)\| \leq d$. Also, for the given positive real number $\hat{e}$, there exists $T>0$, such that for any $k \geq T$, we have $\|x(k)-\hat{x}(k)\| \leq \hat{e}$.

Lemma 2 [15]. For the given any real number $c_{1}$, there exist positive real number $e^{*}$ and set $\Omega(\hat{c})=\left\{x \in R^{n_{x}}: x(k)^{\mathrm{T}} P x(k) \leq \hat{c}\right\}$, where $\hat{c}<c_{1}$, such that if $\|x(k)-\hat{x}(k)\| \leq \hat{e}$, with $\hat{e} \in\left(0, e^{*}\right]$, then $\hat{x}(k) \in \Omega(\hat{c}) \Rightarrow x(k) \in \Omega\left(c_{1}\right)$. 


\section{Distributed Output Feedback Model Predictive Control}

This section studies the design of model predictive controller when states are not measured. Since the input constraint is related to the observer states, states constraint is constraints of the real states, and there are some errors between real states and observer states, the observer errors have influence on the future input and states. So the observer states are used in the performance index directly to design the controller. In order to keep the system stable, we adopt infinite horizon model predictive control strategy. Therefore, the optimization problem at time $k$ is as follows:

$$
\begin{aligned}
& \min J(k)=\min \sum_{l=0}^{\infty}\left(\|\hat{x}(k+l \mid k)\|_{Q}^{2}+\|\hat{u}(k+l \mid k)\|_{R}^{2}\right) \\
& \text { s.t. } \hat{x}(k+l+1)=A \hat{x}(k+l \mid k)+B u(k+l \mid k)+F(y(k+l \mid k)-C \hat{x}(k+l \mid k)) \\
& u(k+l \mid k)=K \hat{x}(k+l \mid k) \in U \\
& x(k+l) \in X
\end{aligned}
$$

where $\hat{x}(k+l \mid k)$ is state predictive value. $\hat{u}(k+l \mid k)$ is input predictive value, $l \geq 0 . Q=Q^{\mathrm{T}} \geq 0$ and $R=R^{\mathrm{T}} \geq 0$ are weight coefficient matrices.

The optimization problem decomposes into two parts as

$$
\begin{array}{ll}
J_{1}(k)=\sum_{l=0}^{N-1}\left(\|\hat{x}(k+l \mid k)\|_{Q}^{2}+\|\hat{u}(k+l \mid k)\|_{R}^{2}\right) & \text { s.t. }(11) \sim(13) \\
J_{2}(k)=\sum_{l=N}^{\infty}\left(\|\hat{x}(k+l \mid k)\|_{Q}^{2}+\|\hat{u}(k+l \mid k)\|_{R}^{2}\right) & \text { s.t. }(11) \sim(13)
\end{array}
$$

Suppose the Lyapunov function

$$
\hat{V}(\hat{x}(k+l \mid k))=\hat{x}(k+l \mid k)^{\mathrm{T}} P \hat{x}(k+l \mid k)=\|\hat{x}(k+l \mid k)\|_{P}^{2}
$$

satisfies the stability constraint

$$
\hat{V}(\hat{x}(k+l+1 \mid k))-\hat{V}(\hat{x}(k+l \mid k)) \leq-\left(\|\hat{x}(k+l \mid k)\|_{Q}^{2}+\|\hat{u}(k+l \mid k)\|_{R}^{2}\right)
$$

When the closed loop system is stable,

$$
\hat{x}(\infty \mid k)=0, \quad \hat{V}(\hat{x}(\infty \mid k))=0
$$

Superpose (16) from $l=N$ to $l=\infty$, we get

$$
-\hat{V}(\hat{x}(k+N \mid k))+\hat{V}(\hat{x}(\infty \mid k)) \leq-\sum_{l=N}^{\infty}\left(\|\hat{x}(k+l \mid k)\|_{Q}^{2}+\|\hat{u}(k+l \mid k)\|_{R}^{2}\right)
$$

That is to say

$$
J_{2}(k) \leq \hat{V}(\hat{x}(k+N \mid k))
$$

Therefore, the optimization problem (15) transforms into minimizing $\hat{V}(\hat{x}(k+N \mid k))$, and then the performance (10) transforms into the following performance

$$
J(k)=\sum_{l=0}^{N-1}\left(\|\hat{x}(k+l \mid k)\|_{Q}^{2}+\|\hat{u}(k+l \mid k)\|_{R}^{2}\right)+\|\hat{x}(k+N \mid k)\|_{P}^{2}
$$

We have the following predictive model based on state observer of the subsystem 


$$
\begin{aligned}
\hat{x}_{i}(k+l \mid k)= & f_{i}(k+l \mid k) \\
= & A_{i i} \hat{x}_{i}(k+l-1 \mid k)+B_{i i} \hat{u}_{i}(k+l-1 \mid k)+\sum_{j \neq i} A_{i j} \hat{x}_{j}(k+l-1 \mid k) \\
& +\sum_{j \neq i} B_{i j} \hat{u}_{j}(k+l-1 \mid k)+F_{i}\left(C_{i i} x_{i}(k+l-1 \mid k)+\sum_{j \neq i} C_{i j} x_{j}(k+l-1 \mid k)\right. \\
& \left.-C_{i i} \hat{x}_{i}(k+l-1 \mid k)-\sum_{j \neq i} C_{i j} \hat{x}_{j}(k+l-1 \mid k)\right) \\
= & A_{i i}^{l} \hat{x}_{i}(k)+\sum_{h=1}^{l} A_{i i}^{l-h} B_{i i} \hat{u}_{i}(k+h-1 \mid k)+\sum_{j \neq i} \sum_{h=1}^{l} A_{i i}^{l-h} A_{i j} \hat{x}_{j}(k+l-1 \mid k-1) \\
& +\sum_{j \neq i} \sum_{h=1}^{l} A_{i i}^{l-h} B_{i j} \hat{u}_{j}(k+l-1 \mid k)+\sum_{h=1}^{l} A_{i i}^{l-h} F_{i} C_{i i} e_{i}(k+h-1 \mid k-1) \\
& +\sum_{j \neq i}^{l} \sum_{h=1}^{l} A_{i i}^{l-h} F_{i} C_{i j} e_{j}(k+l-1 \mid k-1)
\end{aligned}
$$

Its partial derivative is

$$
\begin{aligned}
\frac{\partial f_{i}(k+l \mid k)}{\partial \hat{u}_{j}(k+h-1 \mid k)} & =\sum_{h=1}^{l} \frac{\partial f_{i}(k+l \mid k)}{\partial \hat{x}_{j}(k+h \mid k)} \frac{\partial \hat{x}_{j}(k+h \mid k)}{\partial \hat{u}_{j}(k+h-1 \mid k)}+\frac{\partial f_{i}(k+l \mid k)}{\partial \hat{u}_{j}(k+h-1 \mid k)} \\
& =\sum_{h=1}^{l-1} A_{i i}^{l-h-1} A_{i j} B_{j j}+\sum_{h=1}^{l} A_{i i}^{l-h-1} B_{i j}
\end{aligned}
$$

Because the control law of the subsystem affects not only the performance of its own subsystem, but also that of its downstream subsystem, controller $\mathcal{C}_{i}$ optimizes the performance of its own subsystem $\mathcal{S}_{i}$ and downstream subsystem $\mathcal{S}_{j}(j \neq i)$. Here, input and state sequences got at $k-1$ are made as state sequences estimations $\hat{x}_{j}(k+l \mid k)$ of upstream subsystem. Therefore, define the performance of subsystem $\mathcal{S}_{i}$ as

$$
\begin{aligned}
J_{i}(k)= & \sum_{l=0}^{N-1}\left(\left\|\hat{x}_{i}(k+l \mid k)\right\|_{Q_{i}}^{2}+\left\|\hat{u}_{i}(k+l \mid k)\right\|_{R_{i}}^{2}+\sum_{j \neq i}\left\|\hat{x}_{j}(k+l \mid k-1)+\omega_{i} W_{j i}(k+l \mid k)\right\|_{Q_{j}}^{2}\right) \\
& +\left\|\hat{x}_{i}(k+N \mid k)\right\|_{P_{i}}^{2}+\sum_{j \neq i}\left\|\hat{x}_{j}(k+l \mid k-1)+\omega_{i} W_{j i}(k+l \mid k)\right\|_{P_{j}}^{2}
\end{aligned}
$$

where

$$
W_{j i}(k+l \mid k)=A_{j j}^{l-h-1} A_{j i} B_{j i}\left(\hat{u}_{i}(k+l \mid k)-\hat{u}_{i}(k+l \mid k-1)\right)
$$

And in order to improve the convergence of optimization problem, the weighting coefficients are added.

Next, the model predictive control optimization problem of all subsystems in the distributed model predictive control algorithm is shown as:

Problem 1. For subsystem $\mathcal{S}_{i}, \hat{c}$ satisfies Lemma 1 and 2. $\hat{x}_{i}(k), \hat{x}_{-i}(k), \hat{u}_{i}(k+l-1 \mid k-1), \hat{x}_{+i}(k+l \mid k-1)$ and $\hat{x}_{-i}(k+l \mid k-1), \quad l=1,2, \cdots, N$ are known. Seek control sequence $\hat{u}_{i}(k: k+l-1 \mid k)$ to minimize performance:

$$
\min _{u_{i}(k: k+l-1 \mid k)} J_{i}(k)
$$

s.t.

$$
\begin{aligned}
& \sum_{s=0}^{l} \alpha_{l-s}\left\|\hat{x}_{i}(k+s \mid k)-\hat{x}_{i}(k+s \mid k-1)\right\|_{2} \leq \frac{\xi \kappa \hat{c}}{2 m_{1}} \quad l=1,2, \cdots, N-1 \\
& \left\|\hat{x}_{i}(k+N \mid k)-\hat{x}_{i}(k+N \mid k-1)\right\|_{P_{i}} \leq \frac{\kappa \hat{c}}{2} \\
& \left\|\hat{x}_{i}(k+l \mid k)\right\|_{P_{i}}-\left\|\hat{\tilde{x}}_{i}(k+l \mid k)\right\|_{P_{i}} \leq \frac{\hat{c}}{\mu N} \quad l=1,2, \cdots, N
\end{aligned}
$$




$$
\begin{gathered}
\hat{u}_{i}(k+l-1 \mid k) \in U_{i} \\
\hat{x}_{i}(k+N \mid k) \in \Omega_{i}\left(\frac{\hat{c}}{2}\right)
\end{gathered}
$$

where,

$$
\begin{gathered}
m_{1}=\max _{i}\left\{\text { number of elements in } \mathcal{P}_{i}\right\} \neq 0 \\
\alpha_{l}=\max _{i} \max _{j}\left\{\lambda_{\max }^{\frac{1}{2}}\left(\bar{A}_{i}^{l} A_{i j}\right)^{\mathrm{T}} P_{j} \bar{A}_{i}^{l} A_{i j}\right\} \quad l=0,1, \cdots, N-1
\end{gathered}
$$

$\mathcal{P}_{i}$ is the neighbouring subsystem of the subsystem $\mathcal{S}_{i}$, and $0<\kappa<1,0<\xi \leq 1, \mu>0$ are the design parameters. $\hat{\tilde{x}}_{i}(k+l \mid k)$ is the state track under the action of $\hat{u}_{i}(k+l-1 \mid k-1)$,

$\hat{u}_{i}(k+N-1 \mid k)=K_{i} \hat{x}_{i}(k+N-1 \mid k)$ and $\hat{x}_{j}(k+1: k+N-1 \mid k-1), \quad j \neq i, \quad l=1,2, \cdots, N-1$. Let

$\hat{x}_{i}(k+N \mid k-1)=A_{d i} \hat{x}_{i}(k+N-1 \mid k-1), \quad \bar{A}_{i}=A_{i i}$. In order to guarantee the feasibility, we define the terminal constraint set as $\Omega_{i}\left(\frac{\hat{c}_{1}}{2}\right)$, not $\Omega_{i}\left(\hat{c}_{1}\right)$.

Give the following assumption:

Assumption (ii). At initial moment $k_{0}$, there always exists a feasible control law $\hat{u}_{i}\left(k_{0}+l \mid k_{0}\right) \in U_{i}$, $l=1,2, \cdots, N-1$ of all subsyetems $\mathcal{S}_{i}$ to make observer states $\hat{x}_{i}\left(N+k_{0} \mid k_{0}\right) \in \Omega_{i}\left(\frac{\hat{c}}{2}\right)$, and $\tilde{J}_{i}\left(k_{0}\right)$ bounded.

\section{Performance Analysis}

The distributed model predictive controller based on Lyapunov function and state observer is designed on the condition of initial feasibility, so the main content in this section is to ensure successive feasibility and stability.

\subsection{Successive Feasibility}

This part mainly studies: if the system is feasible at time $k-1(k>1)$, then $\hat{u}_{i}(k: k+N-1 \mid k)=\hat{u}_{i}(k: k+N-1 \mid k-1)$ is the feasible solution of the optimal problem (17)-(22) at time $k$. $\hat{u}_{i}(k: k+N-1 \mid k)$ and $\hat{u}_{i}(k+1: k+N \mid k)$ satisfy the constraint conditions of the problem.

By $\hat{u}_{i}(k: k+N-1 \mid k)=\hat{u}_{i}(k: k+N-1 \mid k-1)$, we get

$$
\left\|\hat{x}_{i}(k+l \mid k)\right\|_{P_{i}}-\left\|\hat{\tilde{x}}_{i}(k+l \mid k)\right\|_{P_{i}}=0
$$

which satisfies the stability condition (20).

Lemma 3. If the Assumption (i) and (ii) are satisfied, and the problem (17)-(22) have feasible solution at any time $k-1$ and satisfy

$$
\hat{x}_{i}(k+N-1 \mid k-2) \in \Omega_{i}\left(\frac{\hat{c}}{2}\right)
$$

$\rho_{i}=\lambda_{\max }\left(A_{d i}^{\mathrm{T}} A_{d i}\right)$, then when

$$
\max _{i}\left(\rho_{i}\right) \leq(1-\kappa)^{2}
$$

we have

$$
\hat{x}_{i}(k+N \mid k-1) \in \Omega_{i}\left(\frac{(1-\kappa) \hat{c}}{2}\right)
$$

Proof. Since the problem (17)-(22) have feasible solution at any time $k-1$, so 


$$
\begin{aligned}
& \left\|\hat{x}_{i}(k+N-1 \mid k-1)\right\|_{P_{i}}-\left\|\hat{x}_{i}(k+N-1 \mid k-2)\right\|_{P_{i}} \\
& \leq\left\|\hat{x}_{i}(k+N-1 \mid k-1)-\hat{x}_{i}(k+N-1 \mid k-2)\right\|_{P_{i}} \leq \frac{\kappa \hat{c}}{2}
\end{aligned}
$$

Therefore,

$$
\left\|\hat{x}_{i}(k+N-1 \mid k-1)\right\|_{P_{i}} \leq\left\|\hat{x}_{i}(k+N-1 \mid k-2)\right\|_{P_{i}}+\frac{\kappa \hat{C}}{2}
$$

From $\hat{x}_{i}(k+N \mid k-1)=A_{d i} \hat{x}_{i}(k+N-1 \mid k-1)$, we derive

$$
\begin{aligned}
\left\|\hat{x}_{i}(k+N \mid k-1)\right\|_{P_{i}} & =\left\|A_{d i} \hat{x}_{i}(k+N-1 \mid k-1)\right\|_{P_{i}} \\
& =A_{d i}^{\mathrm{T}} A_{d i}\left\|\hat{x}_{i}(k+N-1 \mid k-1)\right\|_{P_{i}} \\
& \leq \lambda_{\max }\left(A_{d i}^{\mathrm{T}} A_{d i}\right)\left[\left\|\hat{x}_{i}(k+N-1 \mid k-2)\right\|_{P_{i}}+\frac{\kappa \hat{c}}{2}\right] \\
& \leq(1-\kappa)^{2}\left(\frac{\hat{c}}{2}+\frac{\kappa \hat{c}}{2}\right) \leq \frac{(1-\kappa) \hat{c}}{2}
\end{aligned}
$$

thus

$$
\hat{x}_{i}(k+N \mid k-1) \in \Omega_{i}\left(\frac{(1-\kappa) \hat{c}}{2}\right)
$$

Lemma 4. If the Assumption (i) and (ii) are satisfied, and the problem (17)-(22) have feasible solution at any time $k-1$ and satisfy

$$
\frac{m_{1}}{\lambda_{\min }(P)} \sum_{h=0}^{l} \alpha_{l-h} \leq 1
$$

then for all $l=1,2, \cdots, N$, we have

$$
\left\|\hat{\tilde{x}}_{i}(k+l \mid k)-\hat{x}_{i}(k+l \mid k-1)\right\|_{P_{i}} \leq \frac{\kappa \hat{c}}{2}
$$

and $\hat{x}_{i}(k: k+N-1 \mid k-1)$ satisfies (18) (19).

Proof. When $l=1,2, \cdots, N-1$, since $\hat{u}_{i}(k: k+N-1 \mid k)=\hat{u}_{i}(k: k+N-1 \mid k-1)$, so from the predictive model, we derive

$$
\begin{aligned}
\hat{\tilde{x}}_{i}(k+l \mid k)= & A_{i i}^{l}\left[A_{i i} \hat{x}_{i}(k-1)+B_{i i} \hat{u}_{i}(k-1)+\sum_{j \neq i} A_{i j} \hat{x}_{j}(k-1)+\sum_{j \neq i} B_{i j} \hat{u}_{j}(k-1)\right. \\
& \left.+F_{i}\left(C_{i i} e_{i}(k-1)+\sum_{j \neq i} C_{i j} e_{j}(k-1)\right)\right]+\sum_{h=1}^{l} A_{i i}^{l-h} B_{i i} \hat{u}_{i}(k+h-1 \mid k-1) \\
& +\sum_{j \neq i} \sum_{h=1}^{l} A_{i i}^{l-h} A_{i j} \hat{x}_{j}(k+h-1 \mid k-1)+\sum_{j \neq i} \sum_{h=1}^{l} A_{i i}^{l-h} B_{i j} \hat{u}_{j}(k+h-1 \mid k-1) \\
& +\sum_{h=1}^{l} A_{i i}^{l-h} F_{i} C_{i i} e_{i}(k+h-1 \mid k-1)+\sum_{j \neq i} \sum_{h=1}^{l} A_{i i}^{l-h} F_{i} C_{i j} e_{j}(k+h-1 \mid k-1) \\
\hat{x}_{i}(k+l \mid k-1)= & A_{i i}^{l}\left[A_{i i} \hat{x}_{i}(k-1)+B_{i l} \hat{u}_{i}(k-1)+\sum_{j \neq i} A_{i j} \hat{x}_{j}(k-1 \mid k-2)+\sum_{j \neq i} B_{i j} \hat{u}_{j}(k-1)\right. \\
& +F_{i}\left(C_{i i} e_{i}(k-1)+\sum_{j \neq i} C_{i j} e_{j}(k-1 \mid k-2)\right]+\sum_{h=1}^{l} A_{i i}^{l-h} B_{i i} \hat{u}_{i}(k+h-1 \mid k-1) \\
& +\sum_{j \neq i} \sum_{h=1}^{l} A_{i i}^{l-h} A_{i j} \hat{x}_{j}(k+h-1 \mid k-2)+\sum_{j \neq i} \sum_{h=1}^{l} A_{i i}^{l-h} B_{i j} \hat{u}_{j}(k+h-1 \mid k-1) \\
& +\sum_{h=1}^{l} A_{i i}^{l-h} F_{i} C_{i i} e_{i}(k+h-1 \mid k-1)+\sum_{j \neq i} \sum_{h=1}^{l} A_{i i}^{l-h} F_{i} C_{i j} e_{j}(k+h-1 \mid k-2)
\end{aligned}
$$


The above two formulas subtract, we have

$$
\begin{aligned}
\hat{\tilde{x}}_{i}(k+l \mid k)-\hat{x}_{i}(k+l \mid k-1) \\
=\sum_{j \neq i} \sum_{h=0}^{l} A_{i i}^{l-h} A_{i j}\left(\hat{x}_{j}(k+h-1 \mid k-1)-\hat{x}_{j}(k+h-1 \mid k-2)\right) \\
\quad+\sum_{j \neq i} \sum_{h=0}^{l} A_{i i}^{l-h} F_{i} C_{i j}\left(e_{j}(k+h-1 \mid k-1)-e_{j}(k+h-1 \mid k-2)\right)
\end{aligned}
$$

By Theorem 1, there always exists a $T>0$, when $k>T$, we have $e(k) \rightarrow 0$, and the observer state $\hat{x}(k)$ eventually converges to $x(k)$. Therefore,

$$
\begin{aligned}
& \left\|\hat{\tilde{x}}_{i}(k+l \mid k)-\hat{x}_{i}(k+l \mid k-1)\right\|_{P_{i}} \\
& \leq\left\|\sum_{j \neq i h=0}^{l} A_{i i}^{l-h} A_{i j}\left(\hat{x}_{j}(k+h-1 \mid k-1)-\hat{x}_{j}(k+h-1 \mid k-2)\right)\right\|_{P_{i}} \\
& \quad+\left\|\sum_{j \neq i h=0}^{l} A_{i i}^{l-h} F_{i} C_{i j}\left(e_{j}(k+h-1 \mid k-1)-e_{j}(k+h-1 \mid k-2)\right)\right\|_{P_{i}} \\
& \leq m_{1} \sum_{h=0}^{l} \lambda_{\max }^{\frac{1}{2}}\left(A_{i i}^{l-h} A_{i j}\right)^{\mathrm{T}} P_{i} A_{i i}^{l-h} A_{i j}\left\|\hat{x}_{j}(k+h-1 \mid k-1)-\hat{x}_{j}(k+h-1 \mid k-2)\right\|_{2} \\
& \leq m_{1} \sum_{h=0}^{l} \alpha_{l-h}\left\|\hat{x}_{j}(k+h-1 \mid k-1)-\hat{x}_{j}(k+h-1 \mid k-2)\right\|_{2} \leq \frac{\kappa \hat{c}}{2}
\end{aligned}
$$

Obviously, (23) is satisfied.

Furthermore,

$$
\begin{aligned}
& \sum_{h=0}^{l} \alpha_{l-h}\left\|\hat{x}_{i}(k+h \mid k)-\hat{x}_{i}(k+h \mid k-1)\right\|_{2} \\
& \leq \frac{1}{\lambda_{\min }\left(P_{i}\right)} \sum_{h=0}^{l} \alpha_{l-h}\left\|\hat{x}_{i}(k+h \mid k)-\hat{x}_{i}(k+h \mid k-1)\right\|_{P_{i}} \\
& \leq \frac{1}{\lambda_{\min }\left(P_{i}\right)} \sum_{h=0}^{l} \alpha_{l-h} \frac{\kappa \hat{c}}{2} \leq \frac{\xi \kappa \hat{c}}{2 m_{1}}
\end{aligned}
$$

where, $\xi=\frac{m_{1}}{\lambda_{\min }\left(P_{i}\right)} \sum_{h=0}^{l} \alpha_{l-h}, \quad 0<\xi \leq 1$.

Therefore, $\hat{x}_{i}(k+l \mid k)$ satisfies the constraint (18).

When $l=N$,

$$
\begin{aligned}
\hat{\tilde{x}}_{i}(k+N \mid k)= & A_{d i} \hat{\tilde{x}}_{i}(k+N-1 \mid k)+\sum_{j \neq i} A_{i j} \hat{x}_{j}(k+N-1 \mid k-1)+\sum_{j \neq i} B_{i j} \hat{u}_{j}(k+N-1 \mid k-1) \\
& +F_{i}\left(C_{i i} e_{i}(k+N-1 \mid k-1)+\sum_{j \neq i} C_{i j} e_{j}(k+N-1 \mid k-1)\right) \\
\hat{x}_{i}(k+N \mid k-1)= & A_{d i} \hat{x}_{i}(k+N-1 \mid k-1)+\sum_{j \neq i} A_{i j} \hat{x}_{j}(k+N-1 \mid k-1)+\sum_{j \neq i} B_{i j} \hat{u}_{j}(k+N-1 \mid k-1) \\
& +F_{i}\left(C_{i i} e_{i}(k+N-1 \mid k-1)+\sum_{j \neq i} C_{i j} e_{j}(k+N-1 \mid k-1)\right)
\end{aligned}
$$

The above two formulas subtract, we have

$$
\hat{\tilde{x}}_{i}(k+N \mid k)-\hat{x}_{i}(k+N \mid k-1)=A_{d i}\left(\hat{\tilde{x}}_{i}(k+N-1 \mid k)-\hat{x}_{i}(k+N-1 \mid k-1)\right)
$$

Therefore, (23) is satisfied. 
Next we can derive that $\hat{x}_{i}(k+N \mid k)$ satisfies (19).

Lemma 5. If the Assumption (i) and (ii) are satisfied, and the problem (17)-(22) has feasible solution at any time $k-1$, then $\hat{u}_{i}(k+l-1 \mid k-1) \in U_{i}, l=1,2, \cdots, N$.

Proof. Since the problem (17) (22) has feasible solution at any time $k-1$, so

$$
\hat{u}_{i}(k+l-1 \mid k-1) \in U_{i}
$$

$l=1,2, \cdots, N-1$. Then we only need to proof that when $l=N$, we have $\hat{u}_{i}(k+l-1 \mid k-1) \in U_{i}$. By Lemma 3 and 4 , and triangle inequality, we derive

$$
\begin{aligned}
& \left\|\hat{\tilde{x}}_{i}(k+N-1 \mid k)\right\|_{P_{i}} \\
& \leq\left\|\hat{\tilde{x}}_{i}(k+N-1 \mid k)-\hat{x}_{i}(k+N-1 \mid k-1)\right\|_{P_{i}}+\left\|\hat{x}_{i}(k+N-1 \mid k-1)\right\|_{P_{i}} \\
& \leq \frac{\kappa \hat{c}}{2}+\frac{(1-\kappa) \hat{c}}{2}<\hat{c}
\end{aligned}
$$

then, $\hat{x}_{i}(k+N-1 \mid k) \in \Omega_{i}(\hat{c})$.

Therefore, $\hat{u}_{i}(k+N-1 \mid k-1)=K_{i} \hat{x}_{i}(k+N-1 \mid k) \in U_{i}$.

Lemma 6. If the Assumption (i) and (ii) are satisfied, and the problem (17)-(22) has feasible solution at any time $k-1$, then $\hat{\tilde{x}}_{i}(k+N \mid k) \in \Omega_{i}\left(\frac{\hat{c}}{2}\right)$.

Proof. By triangle inequality, we have

$$
\begin{aligned}
& \left\|\hat{\tilde{x}}_{i}(k+N \mid k)\right\|_{P_{i}} \\
& \leq\left\|\hat{\tilde{x}}_{i}(k+N \mid k)-\hat{x}_{i}(k+N \mid k-1)\right\|_{P_{i}}+\left\|\hat{x}_{i}(k+N \mid k-1)\right\|_{P_{i}} \\
& \leq \frac{\kappa \hat{c}}{2}+\frac{(1-\kappa) \hat{c}}{2} \leq \frac{\hat{c}}{2}
\end{aligned}
$$

then, $\hat{\tilde{x}}_{i}(k+N \mid k) \in \Omega_{i}\left(\frac{\hat{c}}{2}\right)$.

Remark 2. According to Lemma 2 to 6, if the assumption (i) and (ii) are satisfied, then $\hat{u}_{i}(k+l-1 \mid k-1), \hat{\tilde{x}}_{i}(k+l \mid k), l=1,2, \cdots, N$ are feasible solution of (17)-(22). Since $\hat{x}_{i}(k+N) \in \Omega_{i}(\hat{c})$, so by Lemma, we can derive that the closed loop system states satisfy $x_{i}(k+N) \in \Omega_{i}\left(c_{1}\right)$.

\subsection{Stability}

Theorem 2. If the Assumption (i) and (ii) are satisfied, the control law satisfies the constraint condition (18)(22), and design parameters $\kappa, \mu$ satisfy the following inequality

$$
\frac{(N-1) \kappa}{2}+\frac{1}{\mu}<\frac{1}{2}
$$

then the system asymptotically stable at the origin.

Proof. When $\hat{x}\left(k_{0}\right)$ gets into $\Omega(\hat{c})$, we adopt state feedback control to make system asymptotically stable. Next, we only need to prove that when $\hat{x}\left(k_{0}\right) \in X \backslash \Omega(\hat{c})$, the system asymptotically stable to the origin.

Define

$$
V(k)=\sum_{l=1}^{N}\|\hat{x}(k+l \mid k)\|_{P}
$$

By the constraint (20), we have

$$
\|\hat{x}(k+l \mid k)\|_{P}-\|\hat{\tilde{x}}(k+l \mid k)\|_{P} \leq \frac{\hat{c}}{\mu N}
$$




$$
V(k) \leq \sum_{l=1}^{N}\|\hat{\tilde{x}}(k+l \mid k)\|_{P}+\frac{\hat{c}}{\mu}
$$

For $V(k)$, we make difference as

$$
\begin{aligned}
V(k)-V(k-1) \leq & \sum_{l=1}^{N}\|\hat{\tilde{x}}(k+l \mid k)\|_{P}+\frac{\hat{c}}{\mu}-\sum_{l=1}^{N}\|\hat{x}(k+l-1 \mid k-1)\|_{P} \\
& \leq-\|\hat{x}(k \mid k-1)\|_{P}+\frac{\hat{c}}{\mu}+\|\hat{\tilde{x}}(k+N \mid k)\|_{P} \\
& +\sum_{l=1}^{N-1}\left(\|\hat{\tilde{x}}(k+l \mid k)\|_{P}-\|\hat{x}(k+l \mid k-1)\|_{P}\right)
\end{aligned}
$$

Since $\hat{x}(k) \in X \backslash \Omega(\hat{c})$, so

$$
\|\hat{x}(k \mid k-1)\|_{P}>\hat{c}
$$

By Theorem 2, we have

$$
\|\hat{\tilde{x}}(k+N \mid k)\|_{P} \leq \frac{\hat{c}}{2}
$$

By Lemma 4, we have

$$
\sum_{l=1}^{N-1}\left(\|\hat{\tilde{x}}(k+l \mid k)\|_{P}-\|\hat{x}(k+l \mid k-1)\|_{P}\right) \leq \frac{(N-1) \kappa \hat{C}}{2}
$$

Substitute (25)-(27) into (24), we derive

$$
V(k)-V(k-1)<-\hat{c}+\frac{\hat{c}}{\mu}+\frac{\hat{c}}{2}+\frac{(N-1) \kappa \hat{c}}{2}=\hat{c}\left(\frac{(N-1) \kappa}{2}+\frac{1}{\mu}-\frac{1}{2}\right)
$$

therefore, when

$$
\frac{(N-1) \kappa}{2}+\frac{1}{\mu}<\frac{1}{2}
$$

the system is asymptotically stable.

\subsection{Algorithm Steps}

We give the distributed model predictive control algorithm based on Lyapunov function and state observer.

Algorithm Off-line part:

1. Give decay coefficient $\xi$, stable matrix $L$;

2. By Theorem 1, we obtain observer gain $F=\bar{P}^{-1} Y$.

On-line part:

1. Choose the appropriate parameter $Q, P, R$ and Lyapunov function $V=x^{\mathrm{T}}(k) P x(k)$, and obtain the stability domain estimation by calculation (Here is only the form, since the states are unavailable, real to use is $\left.\Omega(\hat{c})=\left\{x \in X: x^{\mathrm{T}}(k) P x(k) \leq \hat{c}, \hat{c}>0\right\}\right)$;

2. Initialize $\hat{x}\left(k_{0}\right), \hat{u}_{i}\left(k_{0}+l \mid k_{0}\right), l=1,2, \cdots, N$, to satisfy Assumption (ii). At $k_{0}$, If $\hat{x}\left(k_{0}\right) \in \Omega(\hat{c})$, then for any $k \geq k_{0}$, adopt feedback control $\hat{u}_{i}(k)=K_{i} \hat{x}_{i}(k)$, or calculate $\hat{x}_{i}\left(k_{0}+l+1 \mid k_{0}\right)$, then send to upstream and downstream subsystems;

3. Receive $\hat{x}_{i}(k), \hat{x}_{j}(k+l \mid k-1), i \neq j$. If $\hat{x}(k) \in \Omega(\hat{c})$, choose the feedback control law $\hat{u}_{i}(k)=$ $K_{i} \hat{x}_{i}(k)$, or solve the optimal problem, we get $\hat{u}_{i}(k: k+N-1 \mid k)$, and then apply $\hat{u}_{i}(k)$ to the subsystem $S_{i}$;

4. Let $k+1 \rightarrow k$, repeat step 2).

\section{Numerical Example}

Consider the distributed system under networked control as follows: 
where:

$$
\begin{gathered}
x(k+1)=A x(k)+B u(k) \\
y(k)=C x(k)
\end{gathered}
$$

$$
A=\left[\begin{array}{ll}
A_{11} & A_{12} \\
A_{21} & A_{22}
\end{array}\right] \quad B=\left[\begin{array}{ll}
B_{11} & B_{12} \\
B_{21} & B_{22}
\end{array}\right] \quad C=\left[\begin{array}{ll}
C_{11} & C_{12} \\
C_{21} & C_{22}
\end{array}\right]
$$

that is this system has two subsystems.

Subsystem 1:

subsystem 2:

$$
\begin{gathered}
x_{1}(k+1)=A_{11} x_{1}(k)+B_{11} u_{1}(k)+A_{12} x_{2}(k)+B_{12} u_{2}(k) \\
y_{1}(k)=C_{11} x_{1}(k)+C_{12} x_{2}(k)
\end{gathered}
$$

where,

$$
\begin{gathered}
x_{2}(k+1)=A_{22} x_{2}(k)+B_{22} u_{2}(k)+A_{21} x_{1}(k)+B_{21} u_{1}(k) \\
y_{2}(k)=C_{22} x_{2}(k)+C_{21} x_{1}(k)
\end{gathered}
$$

$$
\begin{gathered}
A_{11}=\left[\begin{array}{cc}
2.74 & -1.27 \\
2 & 0
\end{array}\right] \quad A_{12}=\left[\begin{array}{cc}
0.97 & 0 \\
0 & 0
\end{array}\right] \quad A_{21}=\left[\begin{array}{cc}
0 & 0.5 \\
0 & 0
\end{array}\right] \quad A_{22}=\left[\begin{array}{cc}
0 & 0 \\
0 & 0.37
\end{array}\right] \\
B_{11}=B_{22}=\left[\begin{array}{c}
0.25 \\
0
\end{array}\right] \quad B_{12}=B_{21}=\left[\begin{array}{c}
0.5 \\
0
\end{array}\right] \quad C_{11}=C_{12}=C_{21}=C_{22}=\left[\begin{array}{ll}
1 & 0
\end{array}\right]
\end{gathered}
$$

Let the subsystem control constraint as $U_{1}=U_{2}=\left\{u_{i} \in \mathcal{R}^{1}:\left|u_{i}\right| \leq 10, i=1,2\right\}$.

We use the Matlab simulationtools to simulate the algorithm proposed in this paper:

By the algorithm above, we can obtain that the stability domain of the subsystem 1 and 2 shown in Figure 2, Figure 3. Choose the initial states $x_{1}=[-2,2], x_{2}=[-2,2]$, the states track of the subsystem 1 and 2 are shown in Figures 4-7, “-” and “*” are real states and estimation states, respectively. Figure 8, Figure 9 show the input track of all the subsystems.

From the simulation results, we can see the algorithm can guarantee that estimation stats track the real states well, and asymptotically stable to the origin. We can also see that the control low satisfied the constraint and stable eventually.

\section{Conclusion}

For a kind of the distributed systems with input and state constraint and unavailable states under networked con-

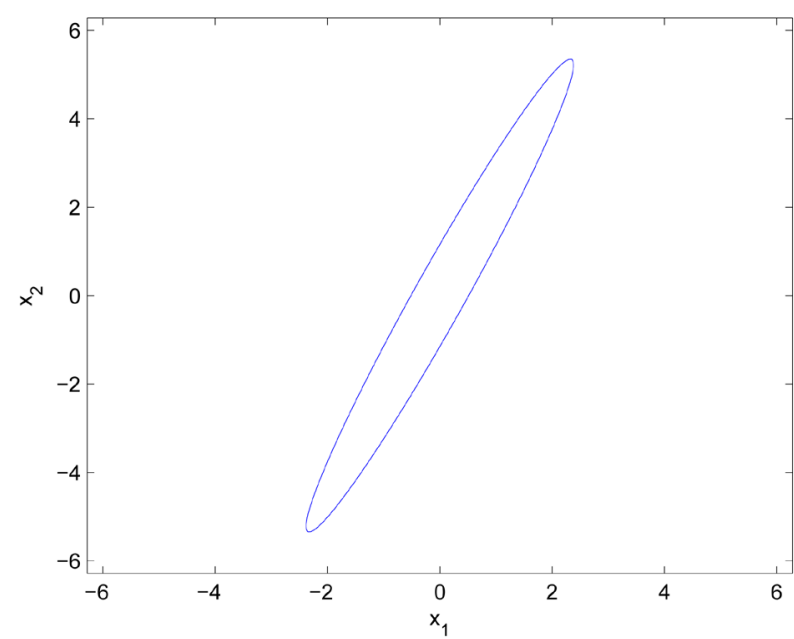

Figure 2. The stability domain of the subsystem 1. 


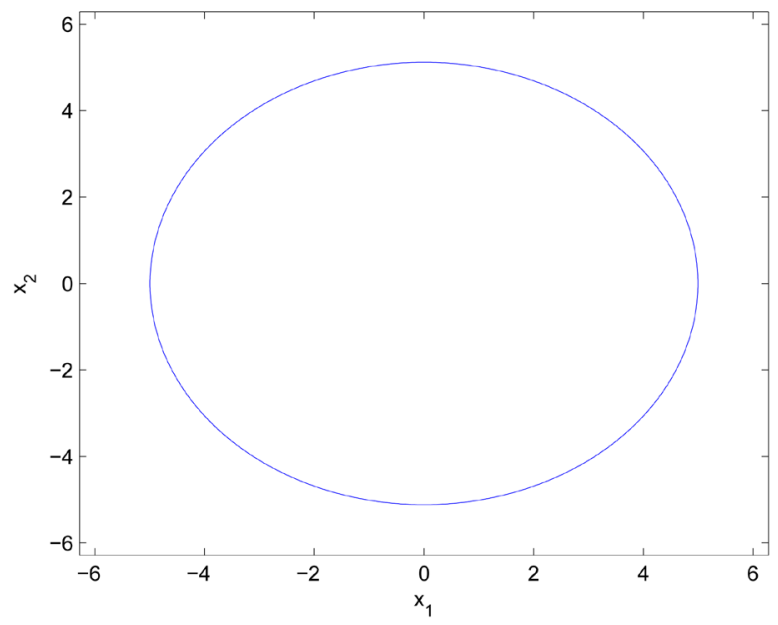

Figure 3. The stability domain of the subsystem 2 .

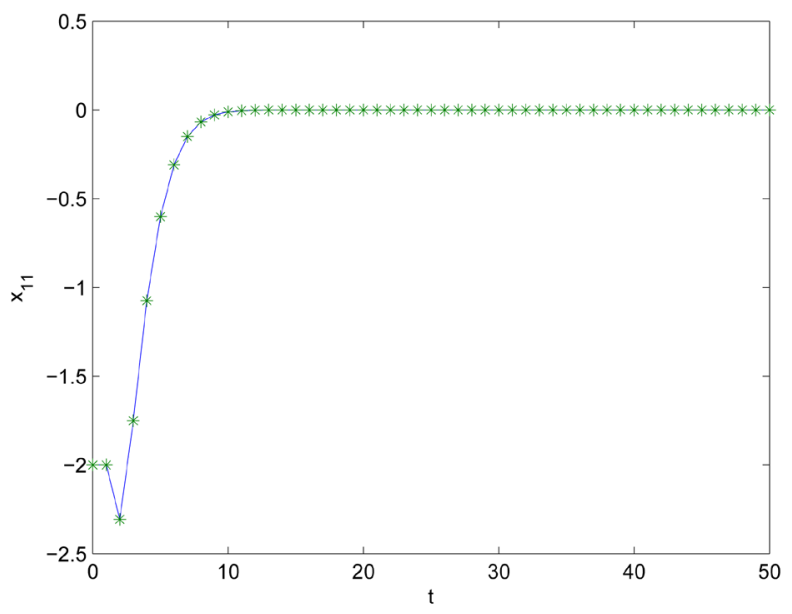

Figure 4. The state components of the subsystem $1 x_{11}$ (“-” representatives the real state, “*” representatives the estimation state $\left.\hat{x}_{11}\right)$.

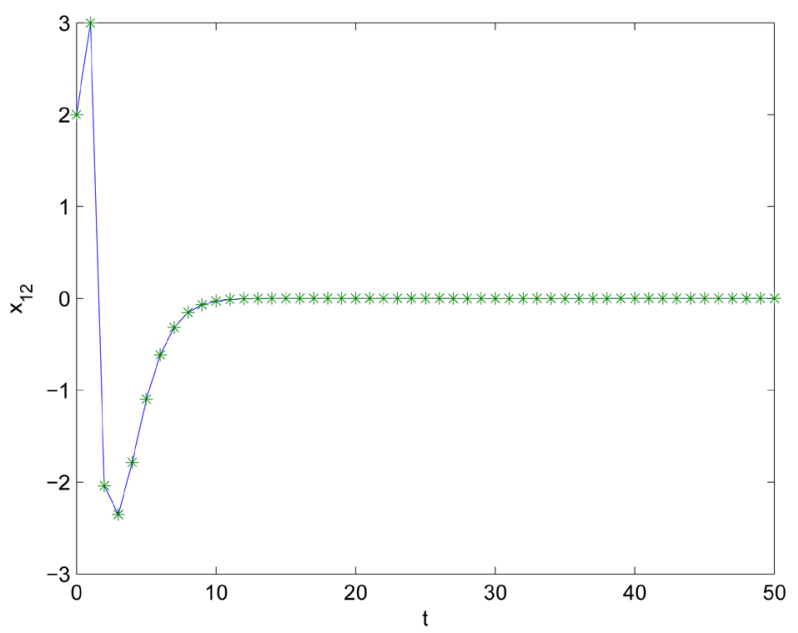

Figure 5. The state components of the subsystem $1 \quad x_{12}$ (“-” representatives the real state, “*” representatives the estimation state $\hat{x}_{12}$ ). 


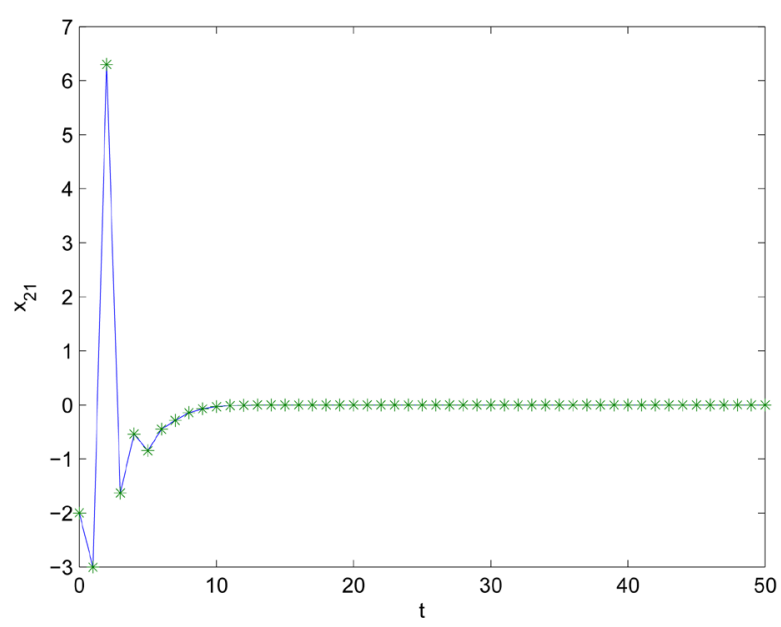

Figure 6. The state components of the subsystem $2 x_{21}$ (“-” representatives the real state, “*” representatives the estimation state $\hat{x}_{21}$ ).

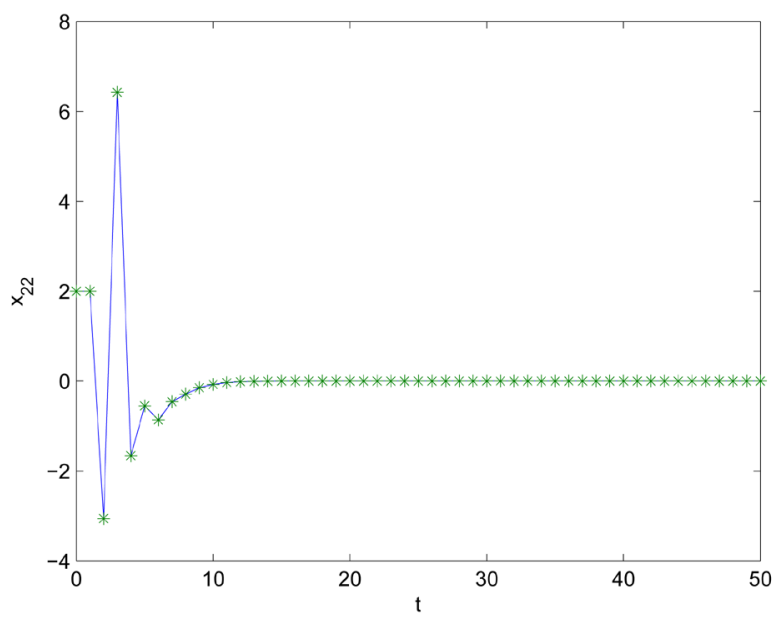

Figure 7. The state components of the subsystem $2 x_{22}$ (“-” representatives the real state, “*” representatives the estimation state $\hat{x}_{22}$ ).

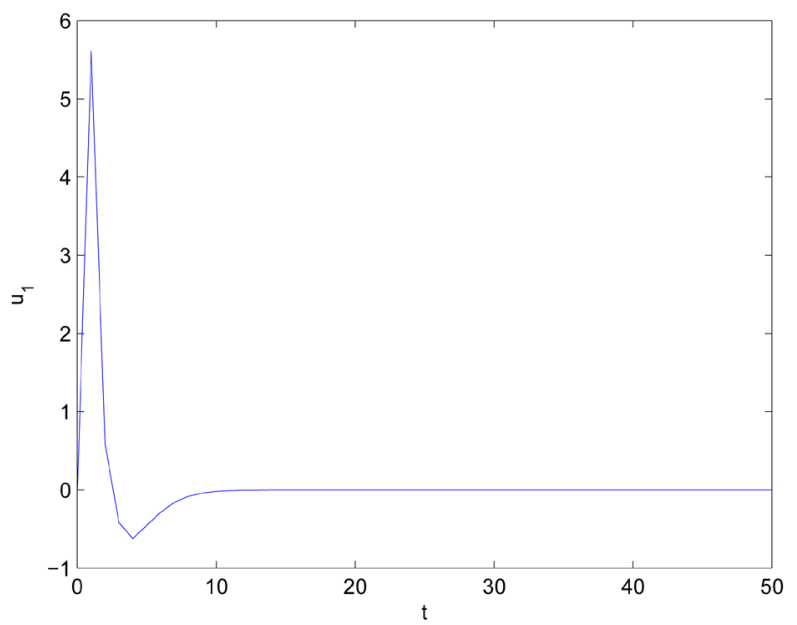

Figure 8 . The control line of the subsystem 1. 


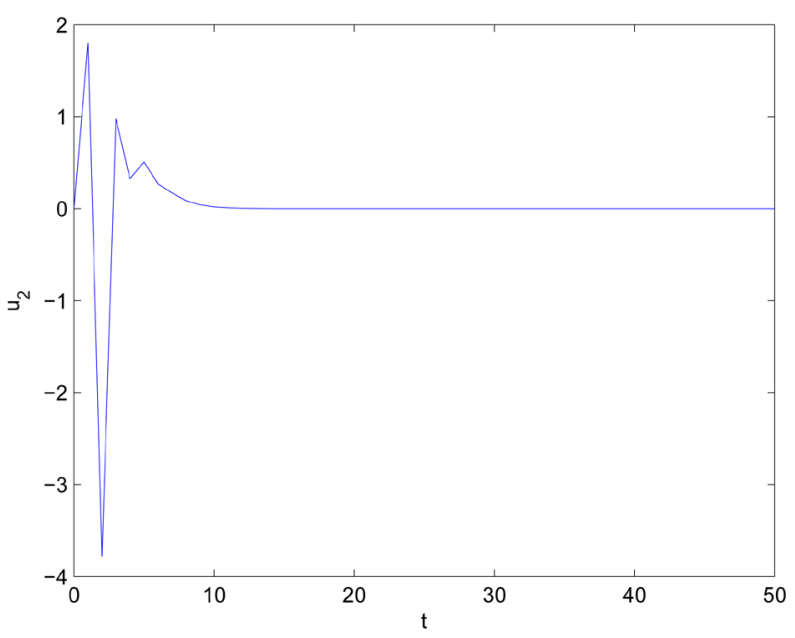

Figure 9. The control line of the subsystem 2.

trol patten, we consider the design and stability problem of the output feedback predictive controller based on Lyapunov function and state observer. The main idea is: For the considered system, use Lyapunov function and states reconstruction to design output feedback controller in order to get the stability domain. Furthermore, the stability domain as a terminal constraint, the distributed model predictive controller is designed. The controller is successive feasibility under the condition of initial feasibility. The simulation results verify the effectiveness of the method proposed in this paper.

\section{References}

[1] Scattolini, R. (2009) Architectures for Distributed and Hierarchical Model Predictive Control—A Review. Journal of Process Control, 19, 723-731. http://dx.doi.org/10.1016/j.jprocont.2009.02.003

[2] Christofides, P.D, Scattolini, R., Muñoz de la Peña, D. and Liu, J.F. (2013) Distributed Model Predictive Control: A Tutorial Review and Future Research Directions. Computers \& Chemical Engineering, 51, 21-41. http://dx.doi.org/10.1016/j.compchemeng.2012.05.011

[3] Min, H.B., Liu, Z.G., Liu, Y., Wang, S.C. and Yang, Y.L. (2013) Distributed Coordination Control of EulerLagrange System under Switching Network Topology. Acta Automatica Sinica, 39, 1003-1010.

[4] Chai, T.Y., Li, S.Y. and Wang, H. (2013) Modeling and Controlling of Complex Industrial Processes under the Network Information Model. Acta Automatica Sinica, 39, 469-470.

[5] Su, B.L., Li, S.Y. and Zhu, Q.M. (2009) Predictive Control of the Initial Stable Region for Constrained Switched Nonlinear Systems. Science in China Press, 39, 994-1003.

[6] Zhu, J. (2002) Intelligent Predictive Control and Its Application. Zhejiang University Press, Hangzhou.

[7] Kong, X.B. and Liu, X.J. (2013) Nolinear Model Predivtive Control for DFIG-Based Wind Power Generation. Acta Automatica Sinica, 39, 636-643.

[8] Camponogara, E., Jia, D., Krogh, B.H. and Talukdar, S. (2002) Distributed Model Predictive Control. IEEE Control Systems, 22, 44-52. http://dx.doi.org/10.1109/37.980246

[9] Li, S.Y. (2008) Model Predictive Control and Application of Global Operating System. Science Press, Beijing.

[10] Al-Gherwi, W., Budman, H. and Elkamel, A. (2011) A Robust Distributed Model Predictive Control Algorithm. Journal of Process Control, 21, 1127-1137. http://dx.doi.org/10.1016/j.jprocont.2011.07.002

[11] Farina, M. and Scattolini, R. (2012) Distributed Predictive Control: A Non-Cooperative Algorithm with Neighbor-toNeighbor Communication for Linear Systems. Automatica, 48, 1088-1096. http://dx.doi.org/10.1016/j.automatica.2012.03.020

[12] Zheng, Y., Li, S.Y. and Li, N. (2011) Distributed Model Predictive Control over Network Information Exchange for Large-Scale Systems. Control Engineering Practice, 19, 757-769. http://dx.doi.org/10.1016/j.conengprac.2011.04.003

[13] Zheng, Y. and Li, S.Y. (2013) Networked Cooperative Distributed Model Predictive Control for Dynamic Coupling Systems. Acta Automatica Sinica, 39, 1778-1786.

[14] Zhao, M. (2013) Predictive Control Strategy for LPV Systems Based on Offline State Observer. Control Engineering 
of China, 20, 1027-1031.

[15] El-Farra, N.H. and Christofides, P.D. (2003) Bounded Robust Control of Constrained Multivariable Nonlinear Process. Chemical Engineering Science, 58, 3025-3047. http://dx.doi.org/10.1016/S0009-2509(03)00126-X

\section{Submit or recommend next manuscript to SCIRP and we will provide best service for you:}

Accepting pre-submission inquiries through Email, Facebook, Linkedin, Twitter, etc A wide selection of journals (inclusive of 9 subjects, more than 200 journals)

Providing a 24-hour high-quality service

User-friendly online submission system

Fair and swift peer-review system

Efficient typesetting and proofreading procedure

Display of the result of downloads and visits, as well as the number of cited articles

Maximum dissemination of your research work

Submit your manuscript at: http://papersubmission.scirp.org/ 